\title{
GIS-based risk map analysis of Leishmaniasis disease in Isfahan, Iran
}

\begin{abstract}
Prediction of Leishmaniasis risk based on socio-environmental factors and its possible spatial relationships is investigated in a Leishmaniasis endemic area of Isfahan, Iran. The Geographical Information System (GIS) is used to link the spatial and significant socioenvironmental indicators with the disease data. Using fuzzy AHP weighting method five classes of risk categories ranging from ñvery lowò to ñvery highò are identified. Spatial analysis is performed to determine the contribution of several environmental factors to the prevalence of the Leishmaniasis disease, and produce a GIS-based risk map showing the relative disease distribution risk level over the study area. Regression analysis shows that socio-environmental factors (population, vegetation, urban and soil area) have a significant contribution to the incidences of Leishmaniasis $(\mathrm{R} 2=0.968)$ with $96 \%$ of the total variation in the Leishmaniasis incidence. Geographically Weighted Regression (GWR) results illustrate that Soil is the factor with biggest positive influence on disease distribution. On the other hand Urban factor is negative for all sub-districts which indicates that developed areas results in a decrease in the Leishmaniasis occurrence. The final developed risk map illustrates that as one move away from the central urban area, the probability of disease prevalence increases. From GWR analysis results, it can be concluded that any step taken to improve the socio-environmental factors would have favourable effects on reducing Leishmaniasis cases. Moreover, the developed risk map provides valuable information which can be used by the Public Health Department for the planning of precautionary measures and for controlling the spread of Leishmaniasis. It will contribute significantly to the spatial prediction of 5 social risk levels varying from very low to very high based on which it envisage the location of high disease distribution risk and that should be given priority in prevention programs. The analysis also indicates factors that should be considered for different locality to reduce the occurrence of the disease and, as a result, control incidence of Leishmaniasis more effectively.
\end{abstract}

Keyword: Fuzzy sets and logic; Geographic information system; Geographically weighted regression; Multi-criteria analysis; Risk map analysis 